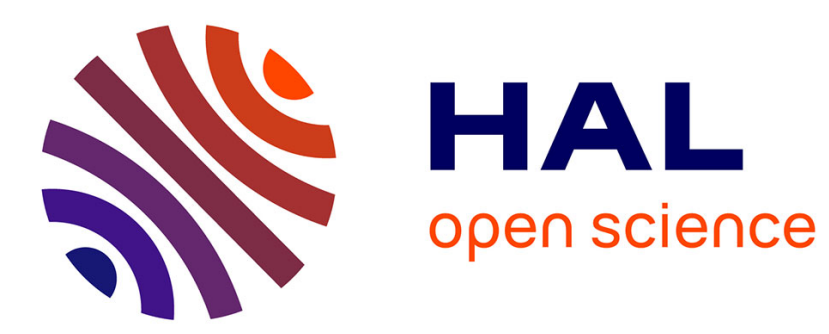

\title{
Statistical Energy Analysis with fuzzy parameters to handle populations of structures
}

\author{
Jean-Louis Guyader, N. Totaro, Laurent Maxit
}

\section{To cite this version:}

Jean-Louis Guyader, N. Totaro, Laurent Maxit. Statistical Energy Analysis with fuzzy parameters to handle populations of structures. Journal of Sound and Vibration, 2016, 379, pp.119 - 134. 10.1016/j.jsv.2016.05.049 . hal-01470460

\section{HAL Id: hal-01470460 https://hal.science/hal-01470460}

Submitted on 12 Nov 2019

HAL is a multi-disciplinary open access archive for the deposit and dissemination of scientific research documents, whether they are published or not. The documents may come from teaching and research institutions in France or abroad, or from public or private research centers.
L'archive ouverte pluridisciplinaire HAL, est destinée au dépôt et à la diffusion de documents scientifiques de niveau recherche, publiés ou non, émanant des établissements d'enseignement et de recherche français ou étrangers, des laboratoires publics ou privés. 


\title{
Statistical Energy Analysis with fuzzy parameters to handle populations of structures
}

\author{
J.-L. Guyader ${ }^{1}$, N. Totaro, L. Maxit \\ Univ Lyon, INSA-Lyon, LVA EA677 \\ 25 bis Avenue Jean Capelle, F-69621 Villeurbanne Cedex, France
}

\begin{abstract}
Statistical modal Energy distribution Analysis (SmEdA) approach was developed to enlarge the application field of Statistical Energy Analysis (SEA) when equipartition of modal energies is not achieved. SmEdA gives more precise results than standard SEA when compared to exact energy response of a deterministic system in the case of low modal overlap, heterogeneous systems or point excitation. The present paper was initiated by this question : when considering a population of similar structures, each of them being described by SmEdA, do the ensemble averaged energies of subsystem and injected power tend to satisfy SEA equations? In other terms, despite the non-equipartition of energy observed on each element of the population of structures does the ensemble averaging leads to SEA equation where equipartition of energy is assumed?

The response to that question that rises from this paper is yes, if the terms of the SEA equation are fuzzy numbers. It results that the energy response given by the model can be interpreted using fuzzy numbers theory.
\end{abstract}

Keywords: Statistical Energy Analysis, Statistical modal Energy distribution Analysis, fuzzy numbers, population of structures

\section{Introduction}

In many practical situations, the vibro-acoustic response of complex structures (i.e. automotive, aircraft, submarine, etc) can be sensitive to small va-

1. Corresponding author. Tel. : + 33172438080. E-mail address : jeanlouis.guyader@insa-lyon.fr 
riations introduced by the manufacturing process specially in the mid and high frequency domains [1]. It is then of interest to develop vibro-acoustic models for estimating statistical characteristics of responses of an ensemble of similar structures defined by random parameters. These statistics can be the mean value, the variance, and eventually higher order statistics like Skewness or Kurtosis [2] and the ensemble of structures can be, for instance, the endproducts of a production line. The models for describing the uncertainties of this ensemble are generally classified in two categories [3] :

(a) The parametric models which consist in identifying some uncertain physical parameters (i.e. geometrical dimensions, Young modulus, thickness variation, etc) and in defining models of uncertainty for these parameters. The vibro-acoustic model then propagates the uncertainties through the dynamic behaviour of the system to give the statistics of the response. A primary approach can be the Monte Carlo technique which can however be time consuming. Alternative approaches like the fuzzy or interval finite element procedures $[4,5]$ have also been developed.

(b) The non-parametric models do not introduce uncertainties in physical parameters but use universal model, independently of the origin of the uncertainties. They are more appropriate for the high frequencies where the number of modes of the system can be significant and their properties (i.e. natural frequencies, mode shapes) can be described by these universal models. In the past, the Poisson natural frequency statistics [6] were considered but they are known now to be valid only for symmetric academic systems like rectangular plates or parallelepiped cavities. The Gaussian Orthogonal Ensemble (GOE) [7, 8, 9] resulting of the random matrix theory has given more accurate results for more complex cases. The non-parametric models of uncertainty are integrated in dedicated vibro-acoustic models. It is the case for the Statistical Energy Analysis (SEA) [10, 11, 12] which is the subject of the present paper. Basically, in SEA, the built-up structure is subdivided into subsystems and the vibration response within each subsystem is characterized by the subsystem energy. For a random broad band excitation in a given frequency band, the energy transfers between the different subsystems are described by the SEA equations. The developments are based on a relation established for two coupled oscillators $p_{i}, p_{j}$ excited by uncorrelated white noise forces :

$$
\pi_{p_{i} p_{j}}=\beta_{p_{i} p_{j}}\left(e_{p_{i}}-e_{p_{j}}\right)
$$


It indicates that the power flow exchanged by the two oscillators $\pi_{p_{i} p_{j}}$ is proportional to the difference of their total energies, $e_{p_{i}}-e_{p_{j}}$. The proportionality coefficient $\beta_{p_{i} p_{j}}$ is called the coupling coefficient. The SEA equations expressing the energy exchanged between multi-modal continuous subsystems have been derived from this basic relation by using simplifying assumptions. In particular, it is assumed that the natural frequencies are uniformly distributed in the frequency band of excitation that constitutes the model of uncertainty in SEA. Although this assumption is strong and can be seen as unrealistic from a practical point of view, it is very useful to justify that the power flow relation established for two coupled oscillators excited by uncorrelated white noise forces can be applied to evaluate the power flow between two coupled modes. The ensemble average of the energy sharing between two modes of two different subsystems are deduced with the supplementary assumption that the difference of energies, $e_{p_{i}}-e_{p_{j}}$ and the coupling coefficient, $\beta_{p_{i} p_{j}}$ are statistically independent,

$$
\left\langle\pi_{p_{i} p_{j}}\right\rangle=\left\langle\beta_{p_{i} p_{j}}\left(e_{p_{i}}-e_{p_{j}}\right)\right\rangle \approx\left\langle\beta_{p_{i} p_{j}}\right\rangle\left(\left\langle e_{p_{i}}\right\rangle-\left\langle e_{p_{j}}\right\rangle\right),
$$

where brackets indicate an ensemble average on the population of structures considered in SEA.

This supplementary assumption has been clearly highlighted and discussed by Mace in a recent paper [13]. In general, it is not respected because $e_{p_{i}}-e_{p_{j}}$ and $\beta_{p_{i} p_{j}}$ are strongly correlated. As a consequence, Eq. (2) should be seen as an approximation.

On another hand, SEA supposes that modal energies into a subsystem are uniformly distributed that is not true in general. Studies (Yap and Woodhouse [14], Fredo [15], Finnveden [16], Mace et al. [17, 18], Ming and Pan [19], Langley et al. [23] and Lafont et al. [20]) illustrate the influence of nonuniformly distributed modal energies on SEA results. When this assumption is not fulfilled, in particular for subsystems with low modal overlap, modern SEA [23] claims to estimate the ensemble mean and variance energy responses of a population of subsystems. Low modal overlap means that the predicted variance becomes large, so that one would not expect the SEA mean to agree very closely with an individual member of the ensemble. This was the key point of our interest in developing SmEdA (Statistical modal Energy distribution Analysis) [25, 26] by writing the coupling of subsytems with the Dual Modal Formulation and suppressing the SEA assumption of equipartition of energy. This approach is based on the knowledge of the modal bases of the 
uncoupled subsystems. By this fact, it is obviously much more time consuming than SEA. However, it proposes a framework to compute explicitly the modal coupling loss factors taking into account geometrical or material complexity of the model. SmEdA, already applied to various industrial structures (car, truck cabin, oil rig, ship), has been developed to better predict energy transmissions of an individual member of the ensemble, in particular in low modal overlap cases that are most often encountered in mechanical structures. However, the consideration of one single deterministic system does not match well with the early developments of SEA which suppose to represent the behaviour of the ensemble average of a population of similar systems. This paper is then initiated by the following question : if a nominal system is well predicted by SmEdA and not by SEA, because equipartition of energy is not achieved, does the effect of ensemble average over a population of structures allows the use of SEA equation (i.e. Eq. (2)) to predict the ensemble average energies of subsystems? This study can be put in relation with previously published works : Starting from the Energy Influence Coefficients [21, 22], Langley and Cotoni [23] derived expressions of the variance of energy for a population of structures. These expressions depend on terms of the standard SEA parameters and additional parameters that describe the variances of the power input and of the coupling between two subsystems. On another hand, Ji and Mace [24] considered two sets of oscillators coupled by springs, they observed that the variance of the excited subsystem depends mainly on the variance of the input power whereas the variance of the receiving subsystem depends on the variance of the intermodal coupling coefficients. The behaviours observed in these two papers $[23,24]$ will be related in the present developments to the modal energy fluctuations in regards of the mean modal energies.

In the present paper, the Dual Modal Formulation and the SmEdA approach are first reminded. Then the ensemble average of a population of structures is studied to see under what conditions SEA equations can be used for ensemble average subsystem energies prediction. It will be shown that SEA equation can be used but Coupling Loss Factors (CLF) and Damping Loss Factors (DLF) have to be defined with uncertainty bounds. Energy response of a population of similar systems can then be estimated using the fuzzy numbers as it will be illustrated on a cavity/plate system. 


\section{Dual Modal Formulation}

The dual modal formulation (DMF) described in [25, 26], is based on a dual displacement-stress formulation of vibroacoustic problems. Two mechanical systems rigidly coupled on a surface $S_{\text {coupling }}$ are considered (see Fig. 1). Subsystem 1 is described by displacement vector $W_{k}(M, t)$ and subsystem 2 by stress tensor $\sigma_{k l}\left(M^{\prime}, t\right)$ where $k, l \in\{1,2,3\}, t$ is time, and $M$ (resp. $M^{\prime}$ ) denotes point of subsystem 1 (resp. subsystem 2). According to the dual modal formulation, subsystem 1 is described by modes of the uncoupledfree subsystem (null stresses on $S_{\text {coupling }}$ ) and subsystem 2 by modes of the uncoupled-blocked subsystem (null displacements on $S_{\text {coupling }}$ ), as shown in Fig. 1. Expanding displacements of subsystem 1 and stresses of subsystem 2, and assuming responses controlled by resonant modes, give :

$$
\begin{gathered}
W_{k}(M, t)=\sum_{p_{1}=1}^{N_{1}} a_{p_{1}}(t) \tilde{W}_{k, p_{1}}(M), \\
\sigma_{k l}\left(M^{\prime}, t\right)=\sum_{p_{2}=1}^{N_{2}} b_{p_{2}}(t) \tilde{\sigma}_{k l, p_{2}}\left(M^{\prime}\right),
\end{gathered}
$$

where

- $a_{p_{1}}(t), b_{p_{2}}(t)$ are modal amplitudes for subsystem 1 and subsystem 2,

- $\tilde{W}_{k, p_{1}}(M)$ are displacement mode shapes of subsystem 1 ,

- $\tilde{\sigma}_{k l, p_{2}}\left(M^{\prime}\right)$ are stress mode shapes of subsystem 2,

- $N_{1}, N_{2}$ are the number of resonant modes of subsystem 1 and subsystem 2 in the frequency band of interest.

With the change of modal variable,

$$
b_{p_{2}}(t)=\dot{c}_{p_{2}}(t)
$$

modal equations of the coupled responses of subsystems write (see [25]) :

$$
\ddot{a}_{p_{1}}(t)+\Delta_{p_{1}} \dot{a}_{p_{1}}(t)+\omega_{p_{1}}^{2} a_{p_{1}}(t)+\frac{1}{M_{p_{1}}} \sum_{m=1}^{N_{2}} \dot{c}_{m}(t) \Psi_{p_{1} m}, \quad \forall p_{1} \in\left[1, \ldots, N_{1}\right],
$$

and

$$
\ddot{c}_{p_{2}}(t)+\Delta_{p_{2}} \dot{c}_{p_{2}}(t)+\omega_{p_{2}}^{2} c_{p_{2}}(t)-\frac{1}{M_{p_{2}} \omega_{p_{2}}^{2}} \sum_{r=1}^{N_{1}} \dot{a}_{r}(t) \Psi_{r p_{2}}, \quad \forall p_{2} \in\left[1, \ldots, N_{2}\right],
$$


where $\Delta_{p_{i}}, \omega_{p_{i}}, M_{p_{i}}$ are modal damping bandwidth, natural angular frequency and modal mass of mode $p_{i}$ of subsystem $i$.

The interaction modal work $\Psi_{p_{1} p_{2}}$ yielded for each couple of modes $\left(p_{1}, p_{2}\right)$ writes :

$$
\Psi_{p_{1} p_{2}}=\int_{S_{\text {coupling }}} \tilde{W}_{k, p_{1}} \tilde{\sigma}_{k l, p_{2}} n_{2, j} \mathrm{~d} S
$$

and $n_{2, j}$ are the components of the outer normal of the volume occupied by subsystem 2.

These equations describe modes interactions with gyroscopic couplings. It is pointed out that a mode of one subsystem is coupled to the modes of the other subsystem but is not directly coupled with the other modes of its own subsystem. This configuration of mode coupling is exactly the one that supposes SEA.

To evaluate the power exchanged by mode $p$ of subsystem 1 with mode $q$ of subsystem 2, one isolates these two modes in the modal equations of motion 6 and 7

$\ddot{a}_{p_{1}}(t)+\Delta_{p_{1}} \dot{a}_{p_{1}}(t)+\omega_{p_{1}}^{2} a_{p_{1}}(t)+\sqrt{\frac{M_{p_{2}} \omega_{p_{2}}^{2}}{M_{p_{1}}}} \gamma_{p_{1} p_{2}} \dot{c}_{p_{2}}(t)=L_{1 p_{1} p_{2}}(t), \quad \forall p_{1} \in\left[1, \ldots, N_{1}\right]$,

and

$\ddot{c}_{p_{2}}(t)+\Delta_{p_{2}} \dot{c}_{p_{2}}(t)+\omega_{p_{2}}^{2} c_{p_{2}}(t)-\sqrt{\frac{M_{p_{1}}}{M_{p_{2}} \omega_{p_{2}}^{2}}} \gamma_{p q} \dot{a}_{p_{1}}(t)=L_{2 p_{1} p_{2}}(t), \quad \forall p_{2} \in\left[1, \ldots, N_{2}\right]$,

where

$$
L_{1 p_{1} p_{2}}(t)=\frac{f_{p_{1}}(t)}{M_{p_{1}}}-\frac{1}{M_{p_{1}}} \sum_{m=1, m \neq p_{2}}^{N_{2}} \dot{c}_{m}(t) \Psi_{p_{1} m}
$$

and

$$
L_{2 p_{1} p_{2}}(t)=\frac{f_{p_{2}}(t)}{M_{p_{2}}}-\frac{1}{M_{p_{2}} \omega_{p_{2}}^{2}} \sum_{r=1, r \neq p_{1}}^{N_{1}} \dot{c}_{r}(t) \Psi_{r^{p_{2}}}
$$

and

$$
\gamma_{p_{1} p_{2}}=\frac{\Psi_{p_{1} p_{2}}}{\sqrt{M_{p_{1}} \omega_{p_{2}}^{2} M_{p_{2}}}} .
$$

Supposing the interaction forces $L_{1 p_{1} p_{2}}(t)$ and $L_{2 p_{1} p_{2}}(t)$ are uncorrelated white noise forces (as done in SEA), the basic SEA relation established by Sharton 
and Lyon [27] can be used :

$$
\pi_{p_{i} p_{j}}=\omega_{c} \eta_{p_{i} p_{j}}\left(e_{p_{i}}-e_{p_{j}}\right)
$$

where $\omega_{c}$ is the central angular frequency of the band of interest, and $\eta_{p q}$ is called the modal coupling loss factor (see [25])). This latter is a function of natural angular frequencies $\omega_{p_{i}}$, modal masses $M_{p_{i}}$, modal bandwidths $\Delta_{p_{i}}$ and interaction modal works $\Psi_{p_{i} p_{j}}$,

$$
\eta_{p_{i} p_{j}}=\frac{\Psi_{p_{i} p_{j}}^{2}}{\omega_{c} M_{p_{i}} \omega_{p_{j}}^{2} M_{p_{j}}}\left(\frac{\Delta_{p_{i}} \omega_{p_{j}}^{2}+\Delta_{p_{j}} \omega_{p}^{2}}{\left(\omega_{p_{i}}^{2}-\omega_{p_{j}}^{2}\right)^{2}+\left(\Delta_{p_{i}}+\Delta_{p_{j}}\right)\left(\Delta_{p_{i}} \omega_{p_{j}}^{2}+\Delta_{p_{j}} \omega_{p_{j}}^{2}\right)}\right) .
$$

\section{SmEdA model}

Let's consider modes $p_{1}$ of subsystem 1 coupled to modes $p_{2}$ of subsystem 2 . In the following, these modes will be denoted as modes $p_{i}$ of subsystem $i(i=\{1,2\})$. The conservation of energy for stationary motion applied to mode $p_{i}$ of subsystem $i$, gives

$$
\pi_{p_{i}}^{\mathrm{inj}}=\pi_{p_{i}}^{\mathrm{diss}}+\sum_{p_{j}=1}^{N_{j}} \pi_{p_{i} p_{j}}, \quad \forall p_{i} \in\left[1, \ldots, N_{i}\right] ; \quad i, j \in\{1,2\} ; \quad j \neq i
$$

where,

- $\pi_{p_{i}}^{\text {inj }}$ is time-averaged power injected into mode $p_{i}$ of subsystem $i$ by the generalized force $f_{p_{i}}$,

- $\pi_{p_{i}}^{\text {diss }}$ is time-averaged dissipated power by internal damping of mode $p_{i}$ of subsystem $i$

- $\sum_{p_{j}=1}^{N_{j}} \pi_{p_{i} p_{j}}$ is time-averaged power flow exchanged by mode $p_{i}$ and modes of subsystem $j$.

The power injected into mode $p_{i}$ by external excitation is, either dissipated by internal damping of the mode or exchanged with modes of subsystem $j$. The power injected in one oscillator excited by a white noise force [27] gives an estimate of $\pi_{p_{i}}^{\mathrm{inj}}$

$$
\pi_{p_{i}}^{\mathrm{inj}}=\frac{\pi}{4 M_{p_{i}}} \bar{S}_{F_{p_{i}}}
$$


where $\bar{S}_{F_{p_{i}}}$ is the power spectral density of the generalised force expressed in $\mathrm{N}^{2} /(\mathrm{rad} / \mathrm{s})$. The power dissipated by internal damping of an oscillator (see [12]) can be related to its total energy by expression

$$
\pi_{p_{i}}^{\mathrm{diss}}=\omega_{p_{i}} \eta_{p_{i}} e_{p_{i}}
$$

where $e_{p_{i}}$ is the time averaged energy of mode $p_{i}$, and $\eta_{p_{i}}$ is the modal damping factor $\left(\Delta_{p_{i}}=\eta_{p_{i}} \omega_{p_{i}}\right)$. Making use of the previous equations, one obtains power balance Eq. (19) for mode $p_{i}$ of subsystem $i$

$$
\pi_{p_{i}}^{\mathrm{inj}}=\eta_{p_{i}} \omega_{p_{i}} e_{p_{i}}+\sum_{p_{j}=1}^{N_{j}} \omega_{c} \eta_{p_{i} p_{j}}\left(e_{p_{i}}-e_{p_{j}}\right), \quad \forall p_{i} \in\left[1, \ldots, N_{i}\right] .
$$

Eq. (19) can be expressed into a linear system of equations

$$
\left\{\begin{array}{c}
\pi_{p_{1}}^{\mathrm{inj}} \\
\ldots \\
\pi_{p_{2}}^{\mathrm{inj}}
\end{array}\right\}=\left[\begin{array}{ccc}
\left(\eta_{p_{1}} \omega_{p_{1}}+\omega_{c} \sum_{p_{2}=1}^{N_{2}} \eta_{p_{1} p_{2}}\right) & \ldots & -\omega_{c} \eta_{p_{1} p_{2}} \\
\ldots & \ldots & \ldots \\
-\omega_{c} \eta_{p_{1} p_{2}} & \cdots & \left(\eta_{p_{2}} \omega_{p_{2}}+\omega_{c} \sum_{p_{1}=1}^{N_{1}} \eta_{p_{1} p_{2}}\right)
\end{array}\right]\left\{\begin{array}{c}
e_{p_{1}} \\
\cdots \\
e_{p_{2}}
\end{array}\right\} .
$$

By solving this system of equations, the modal energies can be obtained and then the total energy of each subsystem can be deduced by adding them

$$
E_{i}=\sum_{p_{i}=1}^{N_{i}} e_{p_{i}}, \quad i \in\{1,2\}
$$

where $E_{i}$ is the time-averaged total energy of subsystem $i$. The model associated to Eq. (20) was called SmEdA (Statistical modal Energy distribution Analysis). It has been applied to complex structures by post-processing finite element method (FEM) [28, 29, 30].

Likewise, the time-averaged total power injected into subsystem $i$ is given by

$$
\Pi_{p_{i}}^{\mathrm{inj}}=\sum_{p_{i}=1}^{N_{i}} \pi_{p_{i}}^{\mathrm{inj}}, \quad i \in\{1,2\} .
$$

\section{Response of a population of similar systems}

\subsection{SmEdA model for each system of the population}

Now let us consider a population of $M$ vibroacoustic systems each of them being constituted of two coupled subsystems. All the vibroacoustic 
systems of the population are similar in the sense that the fluctuations of their characteristics are sufficiently small to permit the description of their energy behaviour by the same type of SmEdA model. In the following a vibroacoustic system of the population is denoted "element of the population" and will be represented by the index $k(\forall k \in[1, \ldots, M])$. The SmEdA modal energy Eq. (19), can be written for each element $k$ of the population

$$
\pi_{p_{i}}^{\mathrm{inj}, \mathrm{k}}=\eta_{p_{i}}^{k} \omega_{p_{i}}^{k} e_{p_{i}}^{k}+\sum_{p_{j}=1}^{N_{j}} \omega_{c} \eta_{p_{i} p_{j}}^{k}\left(e_{p_{i}}^{k}-e_{p_{j}}^{k}\right), \quad \forall p_{i} \in\left[1, \ldots, N_{i}\right] .
$$

\subsection{Ensemble average energies of subsystems}

The response of the population of the $M$ elements can be described by the ensemble average energies $\left\langle E_{i}\right\rangle$ of elements of the population as in SEA. In SEA, the ensemble average energies are related to the ensemble average power $\left\langle\Pi_{i}^{\mathrm{inj}}\right\rangle$ injected in subsystems, where ensemble average is denoted by $\langle\bullet\rangle=\frac{1}{M} \sum_{k=1}^{M} \bullet$. These average quantities can be expressed, using Eqs. (21) to (22), as a function of modal energies and modal injected powers of each element of the population :

$$
\left\langle E_{i}\right\rangle=\frac{1}{M} \sum_{k=1}^{M} \sum_{p_{i}=1}^{N_{i}} e_{p_{i}}^{k}
$$

and

$$
\left\langle\Pi_{i}^{\mathrm{inj}}\right\rangle=\frac{1}{M} \sum_{k=1}^{M} \sum_{p_{i}=1}^{N_{i}} \pi_{p_{i}}^{\mathrm{inj}, k}
$$

The energy of one mode of subsystem $i$ of the element $k$ of the population, can be divided in two parts : the ensemble average of all the modal energies of the population of subsystems $\left\langle e_{i}\right\rangle=\left\langle E_{i}\right\rangle / N_{i}$ plus a fluctuating term. One can write for element $k$ of the population

$$
e_{p_{i}}^{k}=\left\langle e_{i}\right\rangle+\epsilon_{p_{i}}^{k}
$$

It is possible to do the same splitting for the modal injected power

$$
\pi_{p_{i}}^{k}=\left\langle\pi_{i}^{\text {inj }}\right\rangle+\delta_{p_{i}}^{k}
$$


where $\left\langle\pi_{i}^{\mathrm{inj}}\right\rangle=\frac{\left\langle\Pi_{i}^{\mathrm{inj}}\right\rangle}{N_{i}}$ are the ensemble averages of all the modal injected powers of the population of subsystems.

The fluctuating quantities $\epsilon_{p_{i}}^{k}$ and $\delta_{p_{i}}^{k}$ represent the variation of modal energies and injected powers around the ensemble average values. Of course the ensemble average of the modal variations must be equal to 0 , so that

$$
\frac{1}{M} \sum_{k=1}^{M} \sum_{p_{i}=1}^{N_{i}} \epsilon_{p_{i}}^{k}=0
$$

and

$$
\frac{1}{M} \sum_{k=1}^{M} \sum_{p_{i}=1}^{N_{i}} \delta_{p_{i}}^{k}=0
$$

Using the basic Eq. (23), making summation of modal equations for each subsystem and introducing Eqs. (24) to (29), result in two equations relating power injected in subsystems to modal energies of subsystems

$\Pi_{i}^{\mathrm{inj}, \mathrm{k}}+\sum_{p_{i}=1}^{N_{i}} \delta_{p_{i}}^{k}=\omega_{c}\left(\bar{\eta}_{i}^{k}+\bar{\eta}_{i j}^{k}\right) E_{i}^{k}-\omega_{c} \bar{\eta}_{j i}^{k} E_{j}^{k}+\sum_{p_{i}=1}^{N_{i}} \sum_{p_{j}=1}^{N_{j}}\left(\omega_{p_{i}}^{k} \eta_{p_{i}}^{k} \epsilon_{p_{i}}^{k}+\omega_{c} \eta_{i j}^{k}\left(\epsilon_{p_{i}}^{k}-\epsilon_{p_{j}}^{k}\right)\right)$,

with

$$
\bar{\eta}_{i}^{k}=\frac{1}{N_{i}} \sum_{p_{i}=1}^{N_{i}} \frac{\omega_{p_{i}}^{k}}{\omega_{c}} \eta_{p_{i}}^{k}
$$

and

$$
\bar{\eta}_{i j}^{k}=\frac{1}{N_{i}} \sum_{p_{i}=1}^{N_{i}} \sum_{p_{j}=1}^{N_{j}} \eta_{i j}^{k}
$$

The ensemble average of Eq. (30) over the elements of the population is

$$
\begin{aligned}
\left\langle\Pi_{i}^{\mathrm{inj}}\right\rangle= & \omega_{c}\left(\left\langle\bar{\eta}_{i}\right\rangle+\left\langle\bar{\eta}_{i j}\right\rangle\right)\left\langle E_{i}\right\rangle-\omega_{c}\left\langle\bar{\eta}_{i j}\right\rangle\left\langle E_{j}\right\rangle+ \\
& +\sum_{k=1}^{M} \sum_{p_{i}=1}^{N_{i}} \omega_{p_{i}}^{k} \bar{\eta}_{p_{i}}^{k} \epsilon_{p_{i}}^{k}+\sum_{k=1}^{M} \sum_{p_{i}=1}^{N_{i}} \sum_{p_{j}=1}^{N_{j}}\left(\omega_{c} \bar{\eta}_{i j}^{k} \epsilon_{p_{i}}^{k}-\omega_{c} \bar{\eta}_{i j}^{k} p_{j}^{k}\right),
\end{aligned}
$$

The coefficients $\left\langle\bar{\eta}_{i}\right\rangle$ and $\left\langle\bar{\eta}_{i j}\right\rangle$ appear as Damping Loss Factors and Coupling Loss Factors of SEA, resulting from ensemble average of SmEdA modal damping and coupling loss factors. 
The ensemble average eliminates the effect of variation of modal injected powers. Thus, only ensemble average of modal power injected in subsystems appears in the first member of Eq. (33). On the contrary, the variations of modal energies still appear in these equations. As a consequence the standard SEA equation assuming modal equipartition of energy that appears in the first part of the second member is modified by modal energy variations.

It is however possible to write Eq. (33) in a standard SEA form by including modified CLF and DLF through $\langle\beta\rangle$ correcting terms

$$
\left\langle\Pi_{i}^{\mathrm{inj}}\right\rangle=\omega_{c}\left\langle\bar{\eta}_{i}^{\mathrm{mod}}\right\rangle\left\langle E_{i}\right\rangle+\omega_{c}\left(\left\langle\bar{\eta}_{i j}^{\mathrm{mod}}\right\rangle\left\langle E_{i}\right\rangle-\left\langle\bar{\eta}_{j i}^{\bmod }\right\rangle\left\langle E_{j}\right\rangle\right)
$$

with

$$
\begin{gathered}
\left\langle\bar{\eta}_{i}^{\text {mod }}\right\rangle=\frac{1}{M} \sum_{k=1}^{M}\left(\bar{\eta}_{i}^{k}+\beta_{i}^{k}\right)=\left\langle\bar{\eta}_{i}\right\rangle+\left\langle\beta_{i}\right\rangle, \\
\left\langle\bar{\eta}_{i j}^{\text {mod }}\right\rangle=\frac{1}{M} \sum_{k=1}^{M}\left(\bar{\eta}_{i j}^{k}+\beta_{i j}^{k}\right)=\left\langle\bar{\eta}_{i j}\right\rangle+\left\langle\beta_{i j}\right\rangle,
\end{gathered}
$$

where

$$
\left\langle\beta_{i}\right\rangle=\frac{1}{M} \sum_{k=1}^{M} \beta_{i}^{k}=\frac{1}{M} \frac{1}{N_{i}} \sum_{k=1}^{M} \sum_{p_{i}=1}^{N_{i}} \eta_{p_{i}}^{k} \frac{\omega_{p_{i}}^{k}}{\omega_{c}} \frac{\epsilon_{p_{i}}^{k}}{\left\langle e_{i}\right\rangle},
$$

and

$$
\left\langle\beta_{i j}\right\rangle=\frac{1}{M} \sum_{k=1}^{M} \beta_{i j}^{k}=\frac{1}{M} \frac{1}{N_{i}} \sum_{k=1}^{M} \sum_{p_{i}=1}^{N_{i}} \sum_{p_{j}=1}^{N_{j}} \eta_{p_{i} p_{j}}^{k} \frac{\epsilon_{p_{i}}^{k}}{\left\langle e_{i}\right\rangle} .
$$

To summarize, the variation of modal injected power disappears due to the ensemble average contrary to the variation of modal energies that has an influence on the ensemble average energies of subsystems. The validity of standard SEA can be expected if the correcting terms due to modal energies variations remain small.

Two quantities are associated to modal energy variations, the correcting damping loss factors $\left\langle\beta_{i}\right\rangle$ and the correcting coupling loss factors $\left\langle\beta_{i j}\right\rangle$. These correcting terms are small if the relative modal energies variations $\frac{\epsilon_{p_{i}}^{k}}{\left\langle e_{i}\right\rangle}$ are small, that is to say if equipartition is achieved. However, we know that in practical situations this is not the case, in particular for coupled vibroacoustic problem for which the spatial coincidences between the subsystems modes play a significant role. On another hand, correcting terms can also be small and not 
influencing the ensemble average energies of subsystems, even if equipartition is not achieved, when both following conditions are satisfied :

- $\eta_{p_{i}}^{k} \frac{\omega_{p_{i}}^{k}}{\omega_{c}}$ tends to be constant whatever the mode (this condition is derived from Eqs. (37) considering Eq. (28)) and

- $\eta_{p_{i} p_{j}}^{k}$ is not varying for the different couples of subsystems modes (this condition is derived from Eqs. (38) considering Eq. (28))

In general the previous conditions are not achieved and the correcting terms associated to modal energies variations modify standard SEA equation. The estimation of the $\langle\beta\rangle$ correcting terms needs the knowledge of the modal energies variations. In next section, an estimation of bounds of variation for $\langle\beta\rangle$ is proposed.

\section{Bounds of the correcting term $\langle\beta\rangle$}

First we split the modes of each subsystem in two groups having positive and negative variations of energy relatively to the ensemble average value. Eq. (28) writes

$$
\frac{1}{M}\left(\sum_{\left(p_{i}, k\right) \in C_{i}^{+}} \epsilon_{p_{i}}^{k}-\sum_{\left(p_{i}, k\right) \in C_{i}^{-}}\left|\epsilon_{p_{i}}^{k}\right|\right)=0
$$

where $N\left\{C_{i}^{+}\right\}$(resp. $N\left\{C_{i}^{-}\right\}$) is the number of modes of subsystem $i$, for all elements of the population, having positive (resp. negative) energy variation compared to the ensemble average value.

Let us introduce the ensemble average of positive (resp. negative) modal energy variations

$$
\left\langle\epsilon_{i}^{+}\right\rangle=\frac{1}{N\left\{C_{i}^{+}\right\}} \sum_{\left(p_{i}, k\right) \in C_{i}^{+}} \epsilon_{p_{i}}^{k},
$$

and

$$
\left\langle\epsilon_{i}^{-}\right\rangle=\frac{1}{N\left\{C_{i}^{-}\right\}} \sum_{\left(p_{i}, k\right) \in C_{i}^{-}} \epsilon_{p_{i}}^{k},
$$

These two quantities must satisfy, accordingly to Eq. (39), the relation

$$
\left\langle\epsilon_{i}^{+}\right\rangle=\frac{N\left\{C_{i}^{-}\right\}}{N\left\{C_{i}^{+}\right\}}\left\langle\epsilon_{i}^{-}\right\rangle
$$


It is then possible to express positive variations of modal energies by the ensemble average of positive variations $\left\langle\epsilon_{i}^{+}\right\rangle$and a fluctuation term $\delta\left(\epsilon_{p_{i}}^{k}\right)$. The modal energies $e_{p_{i}}^{k}$ are thus spitted in 3 parts : the ensemble average of modal energies $\left\langle e_{i}\right\rangle$, the ensemble average of positive variations of modal energies $\left\langle\epsilon_{i}^{+}\right\rangle$and the fluctuation term $\delta\left(\epsilon_{p_{i}}^{k}\right)$

$$
e_{p_{i}}^{k}=\left\langle e_{i}\right\rangle+\left\langle\epsilon_{i}^{+}\right\rangle+\delta\left(\epsilon_{p_{i}}^{k}\right) \quad \forall p_{i} \in C_{i}^{+}
$$

In the same way, for negative variations of modal energies is

$$
e_{p_{i}}^{k}=\left\langle e_{i}\right\rangle-\left\langle\epsilon_{i}^{-}\right\rangle+\delta\left(\epsilon_{p_{i}}^{k}\right) \quad \forall p_{i} \in C_{i}^{-}
$$

Fig. 2 illustrates these different terms on a simple example. Using these expressions in Eqs. (37) and (38) and neglecting the fluctuation $\delta\left(\epsilon_{p_{i}}^{k}\right)$ compared to the mean value of modal energies variations, give an estimation of the correcting coupling and damping loss factors

$$
\left\langle\beta_{i}\right\rangle \approx \frac{1}{M} \frac{1}{N_{i}}\left(\sum_{\left(k, p_{i}\right) \in C_{i}^{+}} \eta_{p_{i}}^{k} \frac{\omega_{p_{i}}^{k}}{\omega_{c}} \frac{\left\langle\epsilon_{i}^{+}\right\rangle}{\left\langle e_{i}\right\rangle}-\sum_{\left(k, p_{i}\right) \in C_{i}^{-}} \eta_{p_{i}}^{k} \frac{\omega_{p_{i}}^{k}}{\omega_{c}} \frac{\left\langle\epsilon_{i}^{-}\right\rangle}{\left\langle e_{i}\right\rangle}\right)
$$

and

$$
\left\langle\beta_{i j}\right\rangle \approx \frac{1}{M} \frac{1}{N_{i}} \sum_{p_{j}=1}^{N_{j}}\left(\sum_{\left(k, p_{i}\right) \in C_{i}^{+}} \eta_{p_{i} p_{j}}^{k} \frac{\left\langle\epsilon_{i}^{+}\right\rangle}{\left\langle e_{i}\right\rangle}-\sum_{\left(k, p_{i}\right) \in C_{i}^{-}} \eta_{p_{i} p_{j}}^{k} \frac{\left\langle\epsilon_{i}^{-}\right\rangle}{\left\langle e_{i}\right\rangle}\right),
$$

It is now possible to calculate bounds for correcting terms. As an example we consider $\left\langle\beta_{i j}\right\rangle$. By suppressing the negative terms in Eq. (46) it is clear that Eq. (47) holds

$$
\left\langle\beta_{i j}\right\rangle<=\frac{1}{M} \frac{1}{N_{i}} \frac{\left\langle\epsilon_{i}^{+}\right\rangle}{\left\langle e_{i}\right\rangle} \sum_{p_{j}=1}^{N_{j}} \sum_{\left(k, p_{i}\right) \in C_{i}^{+}} \eta_{p_{i} p_{j}}^{k}
$$

The modal coupling loss factors are positive quantities, thus, we increase the second member of Eq. (47) if all the modes are taken into account instead of limiting the summation to modes of positive modal energy variation, and this leads to

$$
\left\langle\beta_{i j}\right\rangle<=\frac{1}{M} \frac{1}{N_{i}} \frac{\left\langle\epsilon_{i}^{+}\right\rangle}{\left\langle e_{i}\right\rangle} \sum_{k=1}^{M} \sum_{p_{i}=1}^{N_{i}} \sum_{p_{j}=1}^{N_{j}} \eta_{p_{i} p_{j}}^{k}=\frac{\left\langle\epsilon_{i}^{+}\right\rangle}{\left\langle e_{i}\right\rangle}\left\langle\bar{\eta}_{i j}\right\rangle .
$$


Repeating the method for the minimum we ended with the result

$$
\left\langle\beta_{i j}\right\rangle>=-\frac{1}{M} \frac{1}{N_{i}} \frac{\left\langle\epsilon_{i}^{-}\right\rangle}{\left\langle e_{i}\right\rangle} \sum_{k=1}^{M} \sum_{p_{i}=1}^{N_{i}} \sum_{p_{j}=1}^{N_{j}} \eta_{p_{i} p_{j}}^{k}=-\frac{\left\langle\epsilon_{i}^{-}\right\rangle}{\left\langle e_{i}\right\rangle}\left\langle\bar{\eta}_{i j}\right\rangle .
$$

One can summarize the different bounds that can be obtained with this method

$$
-\frac{\left\langle\epsilon_{i}^{-}\right\rangle}{\left\langle e_{i}\right\rangle}\left\langle\bar{\eta}_{i}\right\rangle \leq\left\langle\beta_{i}\right\rangle \leq \frac{\left\langle\epsilon_{i}^{+}\right\rangle}{\left\langle e_{i}\right\rangle}\left\langle\bar{\eta}_{i}\right\rangle
$$

and

$$
-\frac{\left\langle\epsilon_{i}^{-}\right\rangle}{\left\langle e_{i}\right\rangle}\left\langle\bar{\eta}_{i j}\right\rangle \leq\left\langle\beta_{i j}\right\rangle \leq \frac{\left\langle\epsilon_{i}^{+}\right\rangle}{\left\langle e_{i}\right\rangle}\left\langle\bar{\eta}_{i j}\right\rangle .
$$

The interesting point is that the estimations of bounds are proportional to standard SEA ensemble averaged coupling and damping loss factors. These bounds rely on two simple physical parameters describing the modal energy variations in the considered subsystem :

- the ratio of ensemble average of positive variations of modal energies and ensemble average of modal energies,

- the ratio of ensemble average of negative variations of modal energies and ensemble average of modal energies.

In the literature it has been shown that vibrations of coupled substructures are characterized by particular modal coupling : the number of modes in subsystem 1 strongly coupled to modes of subsystem 2 is generally low, meaning that only few modes are controlling the power exchange. They constitute a small group of modes of high energies while the majority of modes have low energies. Thus, it can be deduced from Eq. (42), that in general

$$
\frac{\left\langle\epsilon_{i}^{+}\right\rangle}{\left\langle e_{i}\right\rangle}>>\frac{\left\langle\epsilon_{i}^{-}\right\rangle}{\left\langle e_{i}\right\rangle}
$$

\section{SEA with fuzzy parameters}

\subsection{Basic SEA relation with fuzzy numbers}

Eq. (34) establishes that the behaviour of a population of structures can be described by SEA relations but uncertainties on the different parameters appear in the equation because equipartition of energy is not achieved for each element of the population. This situation can be handled in the scheme 
of fuzzy numbers, considering all terms of the SEA equation as fuzzy numbers to represent uncertain knowledge (see [31, 32]). The corresponding equation is denoted in the following "fuzzy SEA equation" and writes

$$
\left\langle\Pi_{i}^{\mathrm{inj}, \mathrm{fuzz}}\right\rangle=\omega_{c}\left\langle\eta_{i}^{\mathrm{fuzz}}\right\rangle\left\langle E_{i}^{\mathrm{fuzz}}\right\rangle+\omega_{c}\left(\left\langle\eta_{i j}^{\mathrm{fuzz}}\right\rangle\left\langle E_{i}^{\mathrm{fuzz}}\right\rangle-\left\langle\eta_{j i}^{\mathrm{fuzz}}\right\rangle\left\langle E_{j}^{\mathrm{fuzz}}\right\rangle\right),
$$

These fuzzy damping and coupling loss factors correspond to the modified one expressed in Eq. (34) with $\langle\beta\rangle$ correcting terms varying in bounds defined by Eqs. (50) to (51). To give an example of fuzzy numbers we are using, let's consider $\left\langle\eta_{j i}^{\text {fuzz }}\right\rangle$. It is defined by the membership function shown in Fig. 3 , where, for sake of simplicity, we use triangle membership functions of support $A_{0}=\left[\left(1-\frac{\left\langle\epsilon_{i}^{-}\right\rangle}{\left\langle e_{i}\right\rangle}\right)\left\langle\eta_{j i}\right\rangle,\left(1+\frac{\left\langle\epsilon_{i}^{+}\right\rangle}{\left\langle e_{i}\right\rangle}\right)\left\langle\eta_{j i}\right\rangle\right]$. The wider the support of the membership function, the higher the imprecision. For a population of very imprecise elements - but however sufficiently similar to obey to the same SmEdA model - the fuzzy number $\left\langle\eta_{j i}^{\text {fuzz }}\right\rangle$ stays in between uncertainty bounds close to the entire support $A_{0}$. If the population is made of elements of less imprecision, the bounds on $\left\langle\eta_{j i}^{\text {fuzz }}\right\rangle$ can be reduced. This is done by the $\alpha$-cut technique, which reduces the support of the membership function at a lower level of uncertainty. The new fuzzy number has a reduced support $A_{\alpha}$ and its membership function contains all values from $\alpha$ to 1 . The higher the value of $\alpha$, the better the confidence in the parameter. If $\alpha=0$, the disparity of the elements of the population is large, if $\alpha=1$ the population is homogeneous, no uncertainties appear and the coupling loss factor coincides with that of the nominal case, $\left\langle\eta_{j i}^{\text {fuzz }}\right\rangle=\left\langle\eta_{j i}^{\text {mod }}\right\rangle$. In the intermediate stages, the homogeneity of the population increases with $\alpha$ and the fuzzy numbers stay in a smaller interval, up to the nominal value when $\alpha=1$.

\subsection{Physical interpretation of bounds of ensemble average of subsystems energies}

In the previous section, bounds for ensemble average of subsystem energies have been obtained. A physical interpretation of these bounds is now discussed. The ensemble average of energy of subsystem $i$ is obtained from equation

$$
\left\langle E_{i}\right\rangle=\frac{1}{M} \sum_{k=1}^{M} E_{i}^{k},
$$

where $E_{i}^{k}$ is the total energy of subsystem $i$ for the element $k$ of the population. Bounds of the ensemble average energy of subsystem $i$ can be simply 
obtained considering maximum and minimum energy of subsystem $i$ in the population

$$
E_{i}^{\max }=\max \left(E_{i}^{k}\right) \quad k \in[1, \ldots, M],
$$

It is obvious from the definition of the ensemble average energy that it is bounded by $E_{i}^{\max }$ and $E_{i}^{\min }$, that is to say

$$
E_{i}^{\max } \geq\left\langle E_{i}\right\rangle \geq E_{i}^{\min }
$$

This relation indicates that bounds of ensemble average of subsystem energy are associated to extremum values of subsystem energies of the population. When using fuzzy numbers in SEA equation, bounds of ensemble average of subsystems energies can be obtained and SEA with fuzzy parameters not only predict ensemble average energies of the subsystems but also the variation of energies of subsystems in the population.

\subsection{Example of SEA with fuzzy parameters}

Let us consider a population of two coupled subsystems, one of them being excited. With Eq. (53) and setting $\left\langle\Pi_{2}^{\text {inj,fuzz }}\right\rangle=0$, we calculate the ratio of ensemble averaged energies of subsystems

$$
\frac{\left\langle E_{2}^{\text {fuzz }}\right\rangle}{\left\langle E_{1}^{\text {fuzz }}\right\rangle}=\frac{\left\langle\eta_{12}^{\text {fuzz }}\right\rangle}{\left\langle\eta_{2}^{\text {fuzz }}\right\rangle+\left\langle\eta_{21}^{\text {fuzz }}\right\rangle}
$$

To use Eq. (57) some fuzzy arithmetic operations must be done (see [31, 32] for more details on fuzzy arithmetic). One possibility is to use interval arithmetic to carry out the analysis. The membership function is cut horizontally at a finite number of $\alpha$-cut levels between 0 and 1 . The different parameters of Eq. (57) have bounds corresponding to the $\alpha$-cut interval and we can determine maximum and minimum values of $\frac{\left\langle E_{2}^{\text {fuzz }}\right\rangle}{\left\langle E_{1}^{\text {fuzz }}\right\rangle}$ at the considered $\alpha$ level. This information is then used to build the membership function of $\frac{\left\langle E_{2}^{\mathrm{fuzz}}\right\rangle}{\left\langle E_{1}^{\mathrm{fuzz}}\right\rangle}$ described at the different $\alpha$ levels considered. It is assumed here, for sake of simplicity, that all the parameters will be scaled simultaneously with the same scale factor when considering different values for $\alpha$. This choice is not mandatory and different $\alpha$-cut values can be set for each parameter.

To illustrate the result, we consider, as an example, the bounds $\frac{\left\langle\epsilon_{1}^{-}\right\rangle}{\left\langle e_{1}\right\rangle}=0.1$, $\frac{\left\langle\epsilon_{1}^{+}\right\rangle}{\left\langle e_{1}\right\rangle}=0.2, \frac{\left\langle\epsilon_{2}^{-}\right\rangle}{\left\langle e_{2}\right\rangle}=0.3$ and $\frac{\left\langle\epsilon_{2}^{+}\right\rangle}{\left\langle e_{2}\right\rangle}=1.5$, that is to say that subsystem 2 is 
supposed to have modal energies varying a lot resulting in a highly fuzzy ensemble average behaviour. Subsystem 1 is supposed to have slightly varying modal energies and in consequence has a lightly fuzzy ensemble average behaviour.

The energy transmission from subsystem 1 to subsystem 2 is characterized by the ratio $\frac{\left\langle E_{2}^{\text {fuzz }}\right\rangle}{\left\langle E_{1}^{\text {fuzz }}\right\rangle}$ that is the fuzzy number presented in Fig. 4. This calculation is done (as explained previously) by classical interval arithmetic calculations for different $\alpha$-cuts and grouped to get the membership function of the ratio $\frac{\left\langle E_{2}^{\text {fuzz }}\right\rangle}{\left\langle E_{1}^{\text {fuzz }}\right\rangle}$.

The result presented in Fig. 5 indicates clearly that for small $\alpha$-cuts the energy ratio of the nominal case is higher than the mean value of the bounds of fuzzy energy ratio. Contrary, for large $\alpha$-cuts both values tend to be equal. One can say that when the fuzzier subsystem is receiving, the nominal case overestimates the average energy transmission of the population.

For the same population of coupled subsystems, but when subsystem 2 is excited and subsystem 1 is receiving, the ensemble average of subsystem energies is a very different fuzzy number than the previous one and shows that the same population of subsystems behaves differently depending on the excited subsystem.

Fig. 5 indicates that when the fuzziest subsystem is excited, the nominal case tends to underestimate the average behaviour of the population especially at large level of uncertainty.

\section{Application to the plate/cavity case}

\subsection{Analysis of the population}

To illustrate the behavior of a population of systems, we consider the example of a rectangular simply supported plate coupled to a cavity and excited by a random broad band point force as presented in Fig. 6 . The characteristics of this system, called "nominal" in the following, are listed in Tab. 1.

For this nominal system, viscous damping with constant modal damping bandwidth is considered and the modal bases of the uncoupled subsystems (plate and cavity) are computed between 1130 and $1414 \mathrm{~Hz}(1250 \mathrm{~Hz}$ third octave band). Then, the SmEdA model is built and the modal injected powers due to the point force are computed. Finally, using Eq. (20), the modal 


\begin{tabular}{|c|c|c|c|}
\hline System & Description & Denomination & Nominal value \\
\hline \multirow{5}{*}{ Cavity } & Length $(\mathrm{m})$ & LXC & 1.9 \\
& Width $(\mathrm{m})$ & LYC & 1.7 \\
& Depth $(\mathrm{m})$ & LZC & 1.3 \\
& Density $\left(\mathrm{kg} / \mathrm{m}^{3}\right)$ & $\rho_{0}$ & 1.29 \\
& Sound speed $(\mathrm{m} / \mathrm{s})$ & $c_{0}$ & 340 \\
& Damping ratio & $\eta_{c}$ & 0.005 at $1250 \mathrm{~Hz}$ \\
& Half-power bandwitdh $(\mathrm{rad} / \mathrm{s})$ & $\Delta_{a} p_{2}$ & 39.27 \\
\hline \multirow{5}{*}{ Plate } & Length $(\mathrm{m})$ & $\mathrm{LXP}$ & 1.2 \\
& Width $(\mathrm{m})$ & LYP & 0.9 \\
& Thickness $(\mathrm{mm})$ & $h_{P}$ & 1 \\
& Density $\left(\mathrm{kg} / \mathrm{m}^{3}\right)$ & $\rho_{P}$ & 7800 \\
& Young's modulus $(\mathrm{Pa})$ & $E_{P}$ & $2.1 \mathrm{e} 11$ \\
& Poisson's coefficient & $\nu_{P}$ & 0.3 \\
& X shift $(\mathrm{m})$ & $d X$ & 0.32 \\
& Y shift $(\mathrm{m})$ & $d Y$ & 0.43 \\
& Damping ratio & $\eta_{P}$ & 0.005 at $1250 \mathrm{~Hz}$ \\
& Half-power bandwitdh $(\mathrm{rad} / \mathrm{s})$ & $\Delta_{p_{1}}$ & 39.27 \\
\hline Force & X position $(\mathrm{m})$ & $X F$ & 0.321 \\
& Y position $(\mathrm{m})$ & $Y F$ & 0.365 \\
\hline
\end{tabular}

TABle 1: Parameters of the plate/cavity system under study. 
energies of the subsystems are obtained. In this frequency band, the plate and the cavity have respectively 92 and 701 modes. The modal energies $e_{p_{1}}$ and $e_{p_{2}}$ of the plate and the cavity are shown in Fig. 7(a).

It is important to underline that for the nominal case, the equipartition of modal energies is not achieved neither in the plate nor in the cavity. This is a well-known result for a plate excited by a point force and Fig. 7(a) illustrates that it is also the case for the non-directly excited subsystem. In [20], the assumption of equipartition of modal energies is reputated to be fulfilled for the Rain-on-the-roof excitations combined with a damping with constant bandwitdh $\Delta_{p_{i}}$. In the present example, if the point force is replaced by a Rain-of-the-roof excitation, the equipartition of modal energies is clearly achieved for the plate - see Fig. 7 - but not in the cavity for which modal energies still have a huge dispersion (of the same order as for the point force case). As a consequence, unless all the subsystems are excited by Rain-ofthe-roof excitations, the assumption of equipartition of modal energy is not achieved in the non-directly excited subsystems.

Let's consider now a population of $M=1000$ similar plate-cavity systems excited by the point force. All the parameters listed in Tab. 1 (mechanical and geometrical parameters of the subsystems and the applied force) are considered as random variables varying statistically with an uniform distribution around the nominal value and with a percentage of variation $+/-\mathrm{N} \%$. To evaluate the dispersion induced by the uncertainties on ensemble average loss factors, we have performed 31 simulations with increasing percentage of variation from $N=0$ to $N=15$ (step : 0.5 ). Fig. 8 compares the averaged loss factors $\left(\left\langle\bar{\eta}_{i}\right\rangle\right.$ and $\left.\left\langle\bar{\eta}_{i j}\right\rangle\right)$ to the modified ensemble averaged loss facors $\left(\left\langle\bar{\eta}_{i}^{\text {mod }}\right\rangle\right.$ and $\left.\left\langle\bar{\eta}_{i j}^{\text {mod }}\right\rangle\right)$ obtained with Eqs. (35) and (36). The bounds of values of the modified loss factors for $98 \%$ of the members of the population are also plotted. One can notice that, in Figs. 8(a) and 8(b), the ensemble average damping loss factors are identical when taking or not into account the correcting terms $\langle\beta\rangle$. The bounds of the damping loss factors are approximately of triangular shape centered on the prescribed value mainly. The effect of the correcting terms on the ensemble averaged coupling loss factors is important on $\left\langle\bar{\eta}_{21}\right\rangle$ but remains weak on $\left\langle\bar{\eta}_{12}\right\rangle$. The bounds of the coupling loss factors have again a triangular shape. Finally, the ratio of ensemble averaged energies $<E_{2}>/<E_{1}>$ is presented in Fig. 9(a) and compared to SEA prediction using non corrected DLF and CLF, Eqs. (31) and (32). Both results are close even if the effect of the correcting term is strong on the $\eta_{21}$ CLF. The bounds of the ratio for $98 \%$ of the population as well as curves representing $+/-2 \sigma$ 


\begin{tabular}{|c|c|c|c|}
\hline Ratios & Exact & Approximation & Error (\%) \\
\hline$\frac{\left\langle\epsilon_{1}^{-}\right\rangle}{\left\langle e_{1}\right\rangle}$ & 0.7528 & 0.7296 & -3.18 \\
\hline$\frac{\left\langle\epsilon_{1}^{+}\right\rangle}{\left\langle e_{1}\right\rangle}$ & 1.2461 & 1.3045 & 4.48 \\
\hline$\frac{\left\langle\epsilon_{2}^{-}\right\rangle}{\left\langle e_{2}\right\rangle}$ & 0.6064 & 0.5364 & -11.54 \\
\hline$\frac{\left\langle\epsilon_{2}^{+}\right\rangle}{\left\langle e_{2}\right\rangle}$ & 1.3524 & 1.2627 & -6.34 \\
\hline
\end{tabular}

TABLe 2: Approximation of the real ratios using the nominal case only.

(where $\sigma$ is the standard deviation of the ensemble) are also plotted. The bounds of the ratio $\left\langle E_{2}\right\rangle /\left\langle E_{1}\right\rangle$ are of stretched triangular shape (not centered on the nominal case, $\mathrm{N}=0 \%$ ) as confirmed by the skewness of the distribution shown in Fig. 9(b). Skewness is a measure of the asymmetry of the probability distribution of a real-valued random variable about its mean. A negative value (resp. positive value) of skew indicates that the tail on the left (resp. right) side is longer than the right (resp. left) side of the distribution. Modern SEA theory based on the GOE is able to predict variance (equal to squared standard deviation) of the ensemble. However Fig. 9 shows that the spread of the ensemble is slightly asymmetric. One of the aims of the present article is to take also into account this particular spread of the ensemble.

\subsection{Derivation of the fuzzy SEA model in the case of the population of plates coupled to cavities}

To derive the fuzzy SEA model for describing the population behaviour, the only needed informations are the ensemble averages of the CLF and DLF and the ratios $\frac{\left\langle\epsilon_{1}^{-}\right\rangle}{\left\langle e_{1}\right\rangle}, \frac{\left\langle\epsilon_{1}^{+}\right\rangle}{\left\langle e_{1}\right\rangle}, \frac{\left\langle\epsilon_{2}^{-}\right\rangle}{\left\langle e_{2}\right\rangle}$ and $\frac{\left\langle\epsilon_{2}^{+}\right\rangle}{\left\langle e_{2}\right\rangle}$. Of course these quantities are not a priori known and we are going to estimate them from the nominal case. The ensemble average of the CLF and DLF are approximated by the ones of the nominal case and the ratios are estimated from the modal energies distribution of the nominal case.

To verify if this approximation is realistic, the real (obtained on the whole population) and the estimated (obtained on the nominal case only) ratios are compared in Tab. 2 for $N=15 \%$.

As can be seen in Tab. 2, even for a large percentage of variation $(+/-15 \%)$ on all the parameters of the plate/cavity system, the estimation of these 
ratios is highly reliable using the nominal case. As a consequence, only the nominal calculation has to be done with SmEdA to get the population behaviour.

The ensemble average of the energy transmission from the plate to the cavity can then be estimated as a fuzzy number using Eq. (57) and the procedure illustrated in section 6.3. The membership function of this fuzzy energy transmission is given in Fig. 10. In this figure, the membership function is calculated either with the nominal case or with the whole population. As can be seen, there are only slight differences between both showing that the nominal case can be representative of the population as previously supposed. Two vertical lines in Fig. 10(a) represent the real bounds of variation of the whole population for $N=15 \%$. The corresponding $\alpha$-cut is approximately 0.63 indicating that only a part of the membership function is representative of such a percentage of variation. For a higher $N$, these bounds are expected to be larger leading to a lower $\alpha$-cut and conversely. The membership function gives an estimate of the variability of the energy transmission that is, as expected, increasing with the variation in the population (decreasing $\alpha$-cut), but in a different way for lower and higher values (the membership function is not symmetric).

\subsection{Evolution versus frequency}

Finally, the same computation of the membership function $\left\langle E_{2}^{\text {fuzz }}\right\rangle /\left\langle E_{1}^{\text {fuzz }}\right\rangle$ has been done on frequency bands with constant bandwidth $\Delta_{f}=200 \mathrm{~Hz}$ centered on central frequencies $f_{c}$ from $300 \mathrm{~Hz}$ to $2100 \mathrm{~Hz}$ considering each time several $\alpha$-cuts between 0.63 and 1 . These ratios obtained with $\alpha$-cuts determined from the whole population or from the nominal case are presented in Fig. 11. The exact bounds of ratios of the population are also plotted. Around $1400 \mathrm{~Hz}$, the correlation between $\alpha$-cut at $\alpha=0.63$ compared to the bounds of the whole population is good either using information on the whole population (Fig. 11(a)) or the nominal case only (Fig. 11(b)). This configuration corresponds to the one presented in the previous section from which the $\alpha$ value has been set. What is much more interesting is that the correlation is also good for all the frequency bands except at really low frequency where the $\alpha$-cut overestimates the real observed variability. The choice of the $\alpha$-cut is thus not highly dependent of the frequency. Obviously, the choice for the $\alpha$ value is dependent on the percentage of variation of the parameters of the system. 


\section{Conclusion}

In this paper we have shown that a population of similar structures, each of them satisfying SmEdA equations, have an ensemble average behaviour that can be described by SEA equations. However coupling loss factors, damping loss factors as well as energies of subsystems and injected powers must be fuzzy numbers in order to represent uncertain knowledge of these quantities. This uncertain knowledge is linked to equipartition of energy in subsystems ; the more equipartition is achieved for elements of the population the smaller the uncertainty on SEA parameters. In order to estimate the uncertainty, the nominal case can be used to characterize fluctuations of modal energies in subsystems. Then making use of fuzzy numbers theory, one can estimate the behaviour of the population through ensemble average energies of subsystems but also from bounds of subsystem energies. These bounds of variations depend first on the fluctuations of subsystems modal energies and, secondly, on the heterogeneity of the population. The influence of the uncertainties on the subsystems energies can be estimated with $\alpha$-cut of fuzzy numbers. It is assumed here, for sake of simplicity, that all the interval parameters are scaled simultaneously with the same scale factor but different $\alpha$-cut values can be set for each parameter. Illustration of the present approach has been made by comparison with Monte Carlo simulation of a population of plates coupled to cavities.

\section{Acknowledgements}

This work was performed within the framework of the Labex CeLyA of Université de Lyon, operated by the French National Research Agency (ANR-10-LABX-0060/ ANR-11-IDEX-0007).

\section{References}

[1] R.J. Bernhard, The limits of predictability due to manufacturing and environmentally induced uncertainty, Proceedings of Internoise '96, St Albans (UK), (1996) 2867-2872.

[2] R.S. Langley, J. Legault, J. Woodhouse, E. Reynders, On the applicability of the lognormal distribution in random dynamical systems, Journal of Sound and Vibration, 332(13) (2013) 3289-3302. 
[3] A. Cicirello, R.S. Langley, The vibro-acoustic analysis of built-up systems using a hybrid method with parametric and non-parametric uncertainties, Journal of Sound and Vibration, 332(9) (2013) 2165-2178.

[4] D. Moens, D. Vandepitte, A fuzzy finite element procedure for the calculation of uncertain frequency-response functions of damped structures : Part 1- Procedure, Journal of Sound and Vibration, 288(3) (2005) 431462.

[5] D. Moens, M. Hanss, Non-probabilistic finite element analysis for parametric uncertainty treatment in applied mechanics : recent advances, Finite Elements in Analysis and Design, 47 (2011) 4-16.

[6] R.H. Lyon, Statistical Analysis of Power Injection and Response in Structures and Rooms. Journal of the Acoustical Society of America, 45 (1969) 545-565.

[7] J. Legault, R.S. Langley, J. Woodhouse, Physical consequences of a nonparametric uncertainty model in structural dynamics, Journal of Sound and Vibration, 331(25) (2012) 5469-5487.

[8] R.S. Langley, A.W.M. Brown, The ensemble statistics of the bandaveraged energy of a random system, Journal of Sound and Vibration, 275(3-5) (2004) 847-857.

[9] R.S. Langley, A.W.M. Brown, The ensemble statistics of the energy of a random system subjected to harmonic excitation, Journal of Sound and Vibration, 275(3-5) (2004) 823-846.

[10] R.H. Lyon, R.G. Dejong, Theory and Application of Statistical Energy Analysis, Butterworth-Heineman, London, 1995.

[11] C.B. Burroughs, R.W. Fischer, F.R. Kern, An introduction to statistical energy analysis, The Journal of the Acoustical Society of America, 101 (1997) 1779-1789.

[12] E.E. Ungar, Fundamentals of Statistical Energy Analysis of Vibrating Systems, U.S. Air Force AFFDL-TR 66-52, 1996.

[13] B.R. Mace, J. Ji, The statistical energy analysis of coupled sets of oscillators. Proceedings of the Royal Society A, 463(2081) (2007) 1359-1377. 
[14] F.F. Yap, J. Woodhouse, Investigation of damping effects on statistical energy analysis of coupled structures, Journal of Sound and Vibration, 197(3) (1996) 351-371.

[15] C. Frëdo, Statistical energy analysis and the individual case, Ph.D. Thesis, Chalmers University of Technology, Sweden, 1995.

[16] S. Finnveden, Energy flows within a three element structure with statistical description of the design parameters, Proceedings of Internoise, Gothenburg (Sweden), 1990.

[17] B.R. Mace, P.J. Shorter, Irregularity, damping, and coupling strength in SEA, Proceedings of IUTAM symposium on Statistical Energy Analysis, Southampton (UK), 1997.

[18] B.R. Mace, J. Rosenberg, The SEA of two coupled plates : an investigation into the effects of subsystem irregularity, Journal of Sound and Vibration, 212(3) (1998) 395-415.

[19] R.S. Ming, J. Pan, The limitation in the SEA prediction of power transmission and energy distribution, Proceedings of Fifth International Congress on Sound and Vibration, Adelaide (Australia), 1997.

[20] T. Lafont, N. Totaro, A. Le Bot, Review of statistical energy analysis hypotheses in vibroacoustics, Proceedings of the Royal Society A, 470 : 20130515 (2013).

[21] J.L. Guyader, C. Boisson, C. Lesueur, Energy transmission in finite coupled plate, Part I : Theory, Journal of Sound and Vibration, 81(1) (1982) 81-92.

[22] B. Mace, P.J. Shorter, Energy flow models from finite element analysis, Journal of Sound and Vibration, 233(3) (2000) 369-389.

[23] R.S. Langley, V. Cotoni, Response variance prediction in the statistical energy analysis on built-up systems, Journal of the Acoustical Society of America, 115(2) (2005) 706-718.

[24] L. Ji, B.R. Mace, Statistical Energy Analysis modelling of complex structures as coupled sets of oscillators : ensemble mean and variance of energy, Journal of Sound and Vibration, 317(3-5) (2008) 760-780. 
[25] L. Maxit, J.-L. Guyader, Estimation of SEA coupling loss factors using a dual formulation and FEM modal information, part I : theory, part II : numerical applications, Journal of Sound and Vibration, 239(5) (2001) 907-948.

[26] L. Maxit, J.-L. Guyader, Extension of SEA model to subsystems with non-uniform modal energy distribution, Journal of Sound and Vibration, 265(2) (2003) 337-358.

[27] T.D. Scharton, R.H. Lyon, Power flow and energy sharing in random vibration, The Journal of the Acoustical Society of America, 43 (1968) 1332-1343.

[28] N. Totaro, J.L. Guyader, Extension of the Statistical modal Energy distribution Analysis for estimating energy density in coupled subsystems, Journal of Sound and Vibration, 331(13) (2012) 3114-3129.

[29] N. Totaro, C. Dodard, J.L. Guyader, SEA Coupling Loss Factors of complex vibro-acoustic systems, Journal of Vibration and Acoustics, 131(4) (2009) 041009-1.

[30] Y. Gerges, H.D. Hwang, K. Ege, L. Maxit, C. Sandier, Vibroacoustic modeling of a trimmed truck cab in the mid-frequency range, Proceedings of Internoise 2015, San Francisco (USA), 2015.

[31] D. Dubois, H. Prade, Fundamentals of Fuzzy sets, Kluwer Academic Publishers, Boston, 2000.

[32] A. Kaufman, M. M. Gupta, Introduction to Fuzzy Arithmetic, Theory and applications, Van Nostrand Reinhold Co. Inc., Workingham, Berkshire, 1984. 


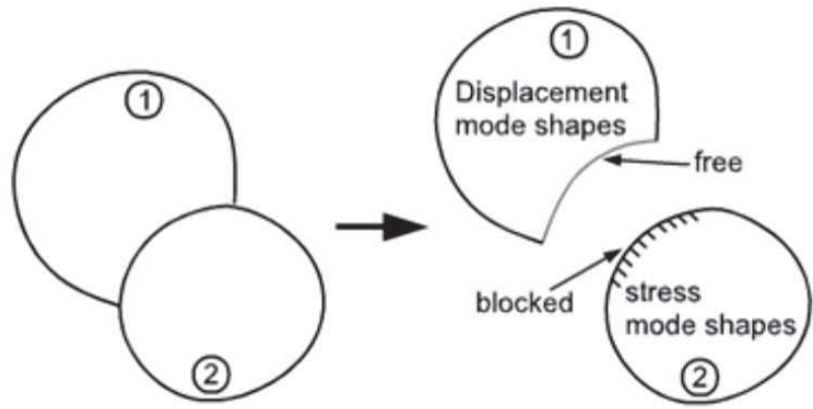

Figure 1: Two coupled mechanical systems 


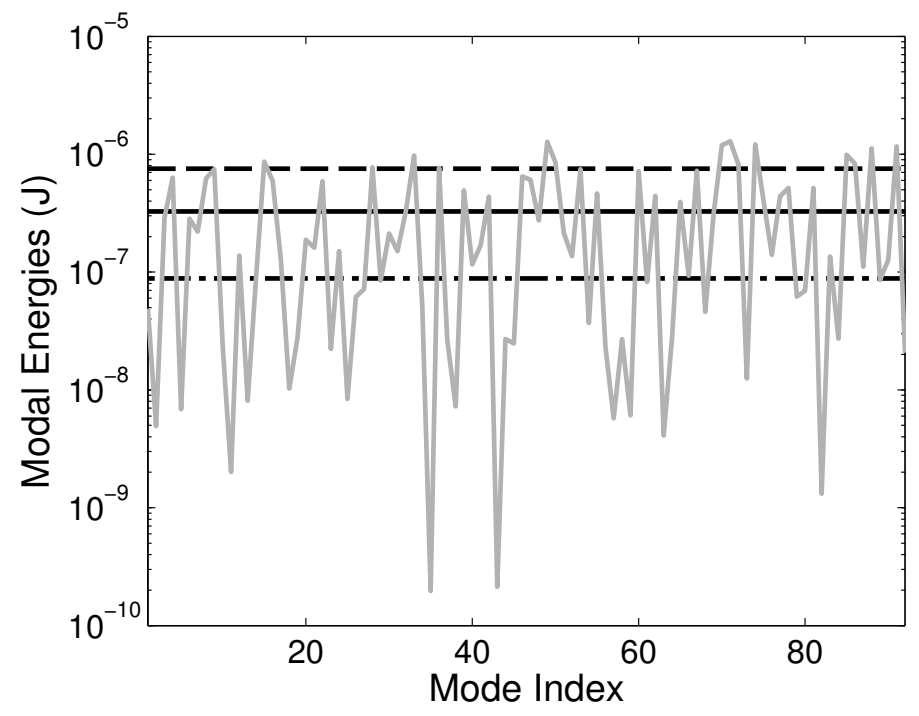

Figure 2: Modal energies for subsystem 1. Solid gray line : modal energies; solid black line : ensemble average of modal energies $\left\langle e_{1}\right\rangle$; dashed line : ensemble average of positive variations of modal energies $\left\langle e_{1}\right\rangle+\left\langle\epsilon_{1}^{+}\right\rangle$; dash-dotted line : ensemble average of negative variations of modal energies $\left\langle e_{1}\right\rangle-\left\langle\epsilon_{1}^{-}\right\rangle$. 


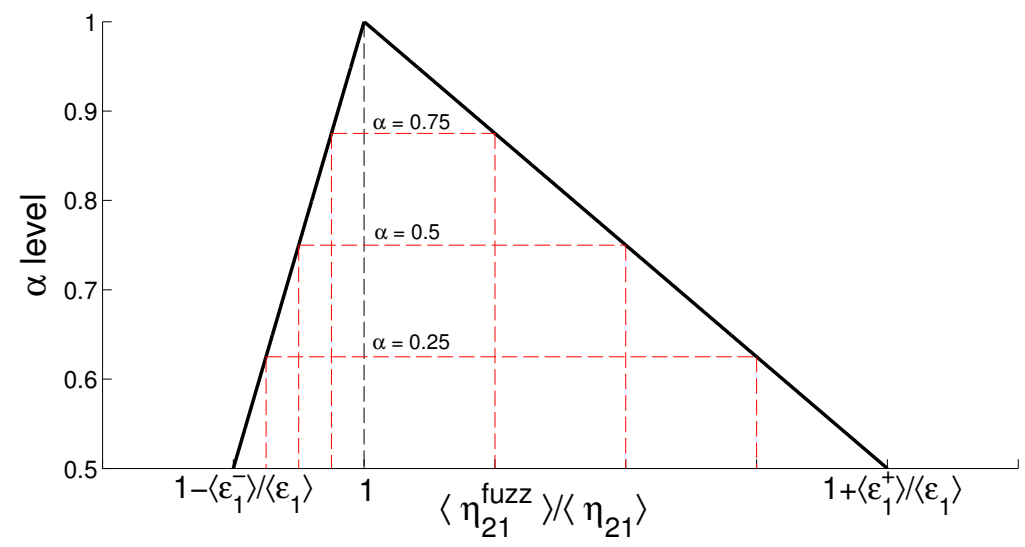

Figure 3: Membership function of the fuzzy number $\frac{\left\langle\eta_{21}^{\text {fuzz }}\right\rangle}{\left\langle\eta_{21}\right\rangle}$ and $\alpha$-cuts. 


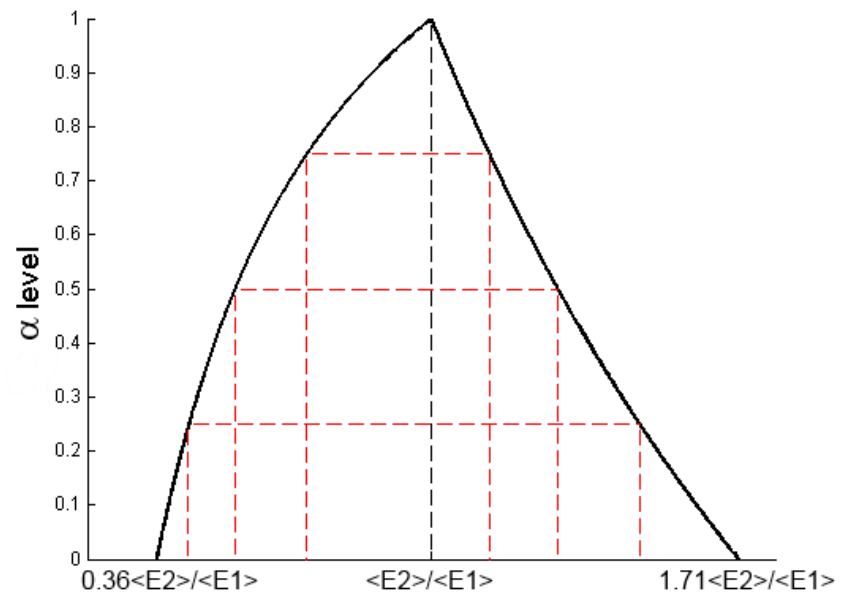

FigURE 4: Membership function of the fuzzy number $\frac{\left\langle E_{2}^{\mathrm{fuzz}}\right\rangle}{\left\langle E_{1}^{\mathrm{fuzz}}\right\rangle}$, subsystem 1 is excited. 


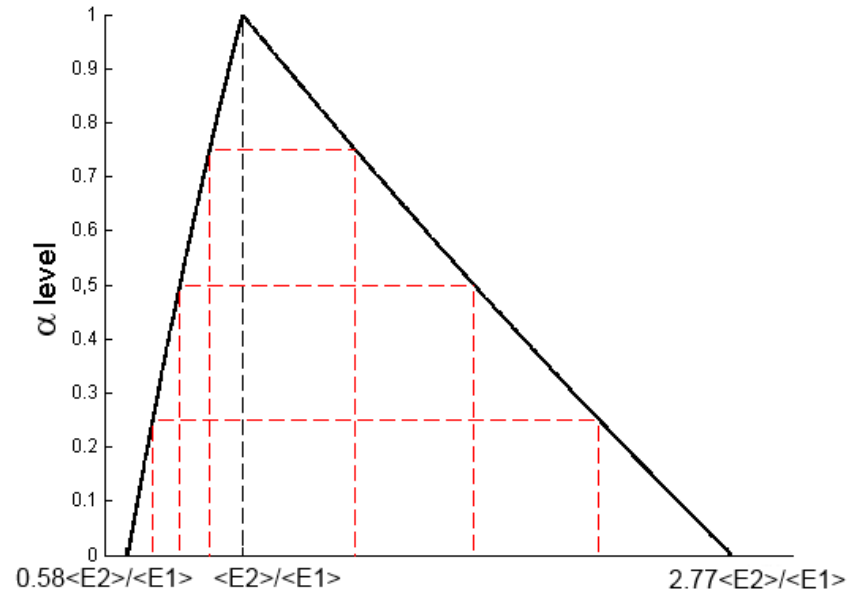

FiguRE 5: Membership function of the fuzzy number $\frac{\left\langle E_{2}^{\text {fuzz }}\right\rangle}{\left\langle E_{1}^{\text {fuzz }}\right\rangle}$, subsystem 2 is excited. 


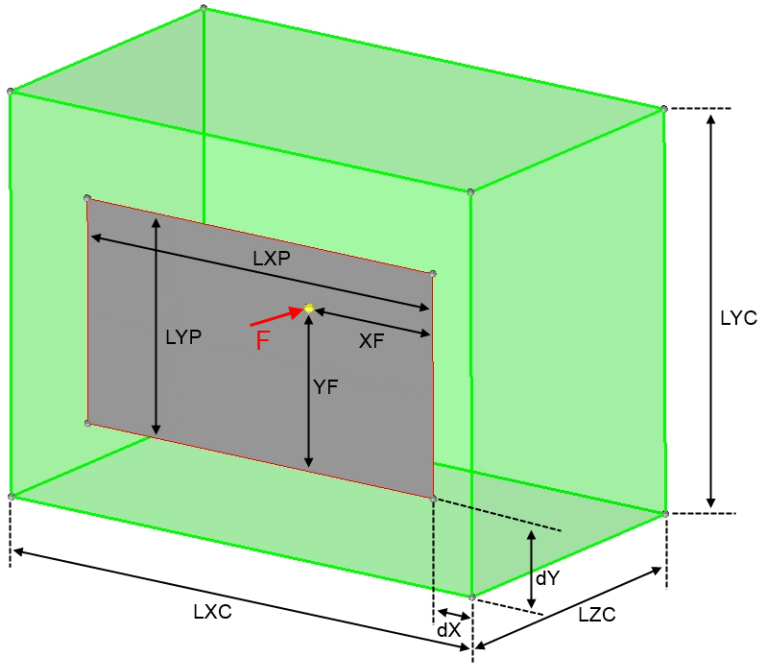

FiguRE 6: A vibrating rectangular plate excited by a point force radiates into a cavity with rigid walls. 


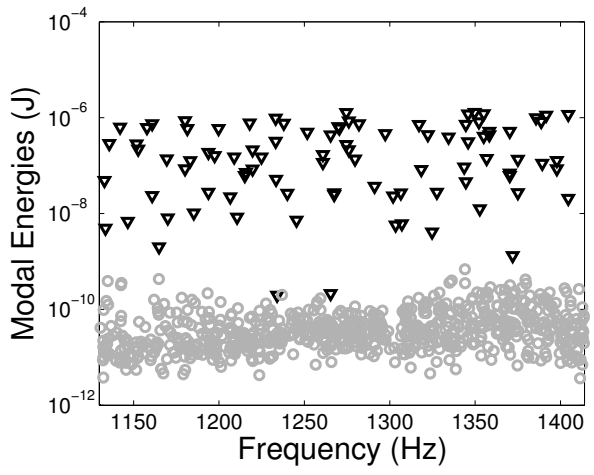

(a)

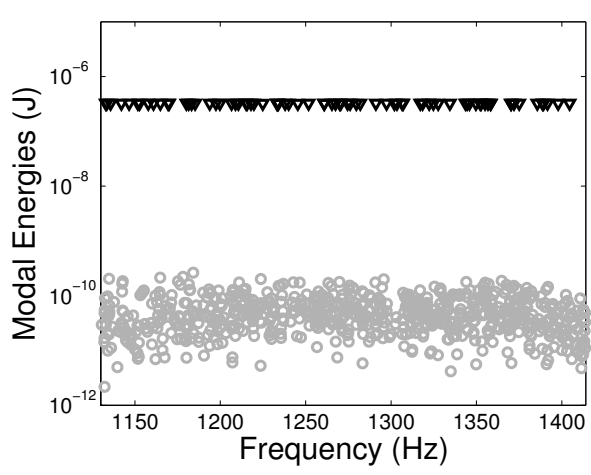

(b)

FiguRE 7: Modal energies of the plate and the cavity for modes in $1250 \mathrm{~Hz}$ third octave band. Black triangles : modal energies $e_{p}$ of the plate; gray circle : modal energies $e_{q}$ of the cavity. (a) Point force excitation; (b) Rain-on-the-roof excitation (equivalent injected power). 


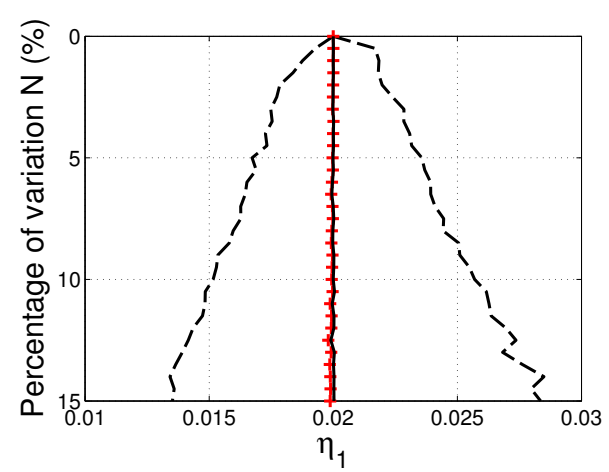

(a)

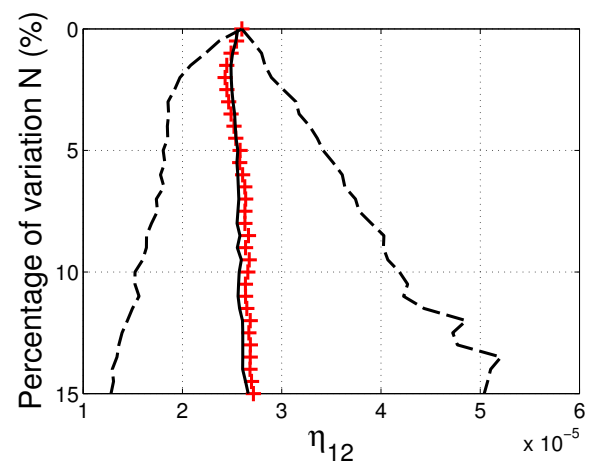

(c)

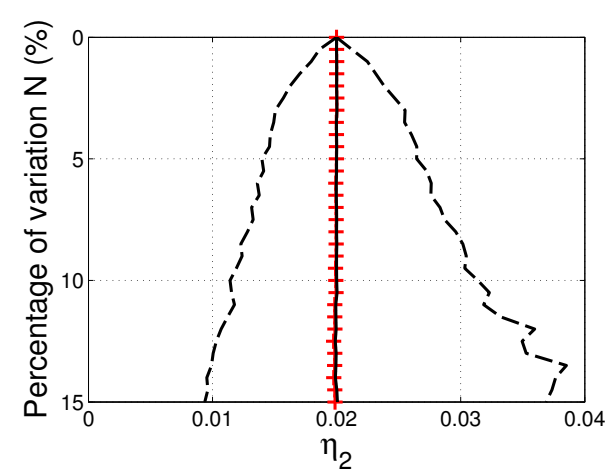

(b)

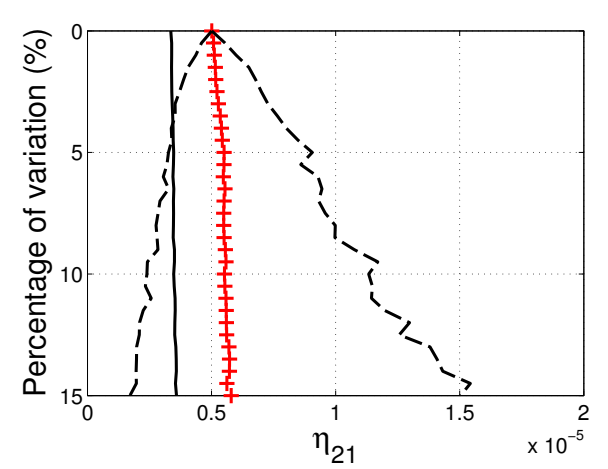

(d)

Figure 8: (a) DLF of the plate;(b) DLF of the cavity; (c) CLF between the plate and the cavity; (d) CLF between the cavity and the plate as a function of N. Black solid lines : ensemble average of loss factors $\left\langle\bar{\eta}_{1}\right\rangle\left\langle\bar{\eta}_{2}\right\rangle,\left\langle\bar{\eta}_{12}\right\rangle$ and $\left\langle\bar{\eta}_{21}\right\rangle$; Red crosses : modified loss factors $\left\langle\bar{\eta}_{1}^{\bmod }\right\rangle,\left\langle\bar{\eta}_{2}^{\bmod }\right\rangle,\left\langle\bar{\eta}_{12}^{\bmod }\right\rangle$ and $\left\langle\bar{\eta}_{21}^{\bmod }\right\rangle$; Dashed black lines : limits values for $98 \%$ of the members of the population of modified loss factors. $1250 \mathrm{~Hz}$ third octave band. 

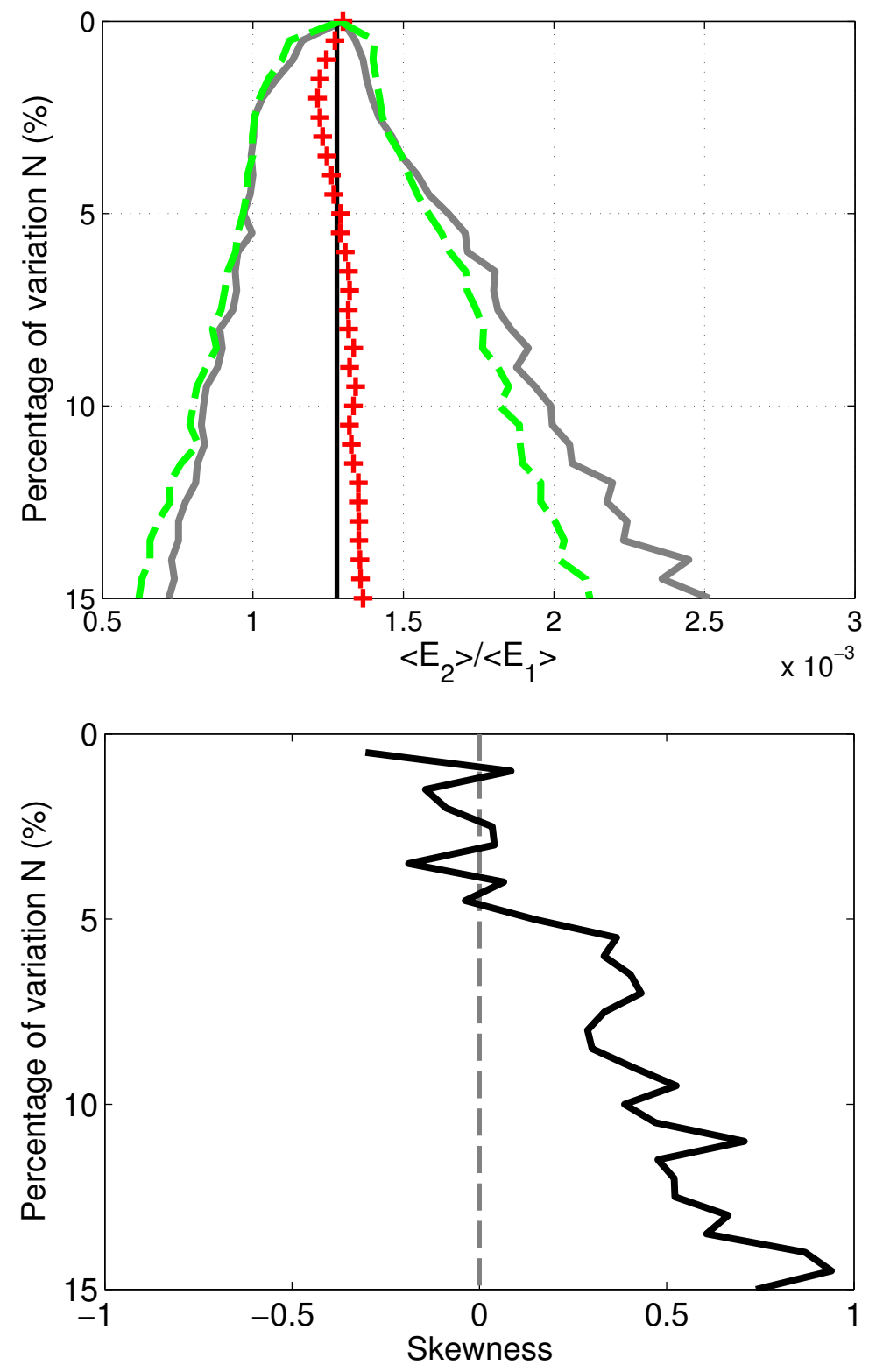

Figure 9: (a) Ratio between energy of the cavity and energy of the plate as a function of N. Black solid lines : SEA prediction; Red crosses : prediction obtained with modified loss factors; Solid gray lines : limits values for $98 \%$ of the members of the population; Dashed green lines : + /- two times the standard deviation of the ensemble. (b) Skewness of energy ratios of the ensemble. A symmetric distribution has a skewness equal to zero (vertical gray line). $1250 \mathrm{~Hz}$ third octave band. 


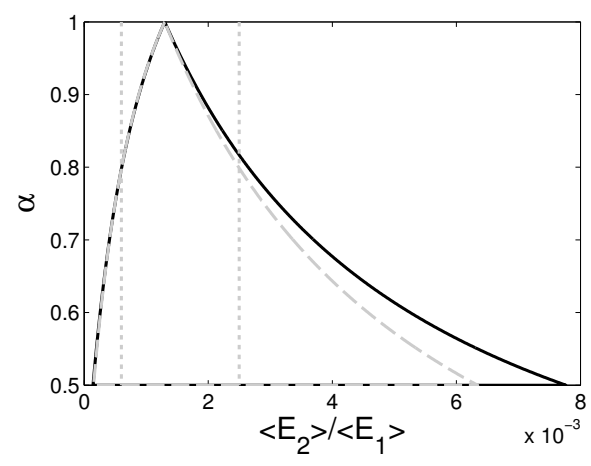

(a)

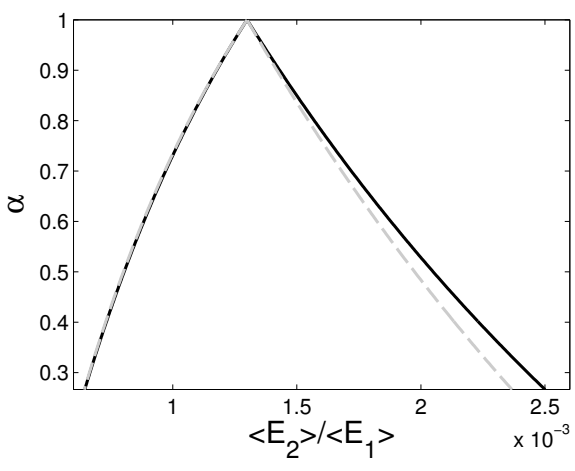

(b)

Figure 10: Membership function of $\left\langle E_{2}^{f u z z}\right\rangle /\left\langle E_{1}^{f u z z}\right\rangle$. Black solid line : estimated using modal energies of the whole population of systems ; gray dashed line : estimated using only the modal energies of the nominal case; Vertical dotted lines : Limits of the energy ratios observed in the population for $\mathrm{N}=15 \%$; Red solid line : RoR. (a) $\alpha \in[0 ; 1]$; (b) zoom for $\alpha \in[0.63 ; 1]$ 


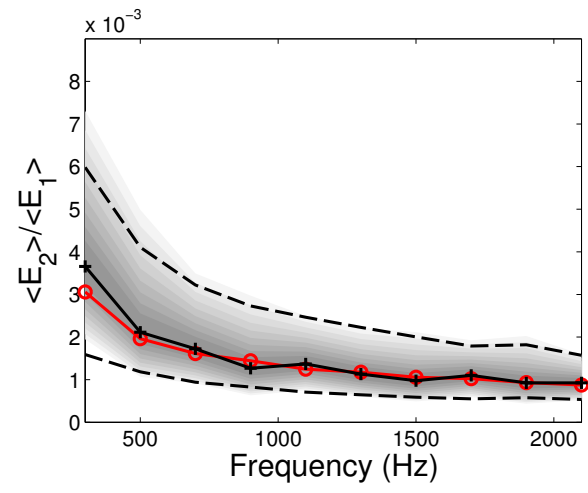

(a)

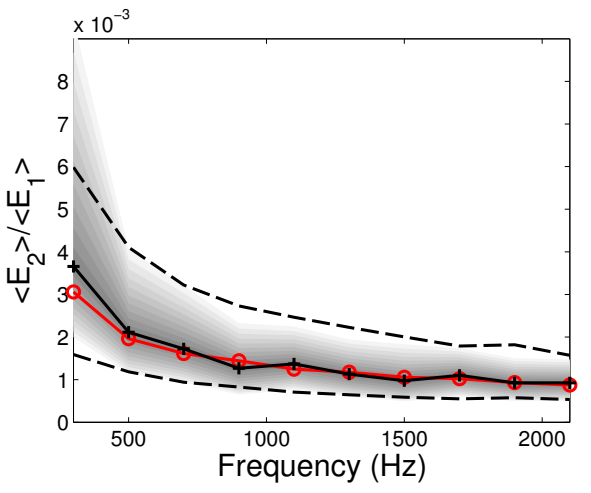

(b)

Figure 11: Evolution of the ratio $\left\langle E_{2}\right\rangle /\left\langle E_{1}\right\rangle$ as a function of frequency. Gray areas : fuzzy ratio with $\alpha$-cuts form 0.63 to 1 obtained from (a) the whole population and (b) the nominal case. Black crosses : ratio obtained with SmEdA modal CLF; red circles : ratio obtained with SEA CLF ; Dashed black lines : limits values for $98 \%$ of the members of the population 